\title{
Handwritten Recognition using Slope and Curvature Functions
}

\author{
Mehdi Yaghoubi \\ Department of Computer Science, Golestan \\ University, 49138-15759 Gorgan, Iran
}

\author{
Soheila Karbasi \\ Department of Computer Science, Golestan \\ University, 49138-15759 Gorgan, Iran
}

\begin{abstract}
Letter recognition and handwritten processing is one of the major and open problems in Artificial Intelligent (AI) domain. This study introduces a method based on statistical and geometrical techniques to recognize handwritten digits and letters. These techniques use the fuzzy logic to create the vector curves. Inputs are online digits or letters and outputs are two arrays of slope and curvature values. The slope and curvature values of training data are stored in a database and used in comparison phase. The test results show that $96.98 \%$ of inputs are correctly recognized.
\end{abstract}

\section{General Terms}

Pattern recognition, Fuzzy logic, Persian digits \& letters

\section{Keywords}

Vector curve, Slope function, Curvature function

\section{INTRODUCTION}

Handwritten recognition in Latin and Chinese languages has been started over the last decades and many commercial packages are created in this field $[1,5]$. Furthermore, similar researches for Persian language have been recently studied which have more complexity and variety [4]. One of the most used techniques to recognize scanned handwritten is fuzzy logic $[3,7]$. In this technique, the input images are narrowed and the principal points of them are specified and saved as the patterns. In narrowing phase, undesirable effect of pen thickness and its pressure is eliminated. Figure 1 shows digit 3 in Arabic-Indic numeral system before and after narrowing phase.

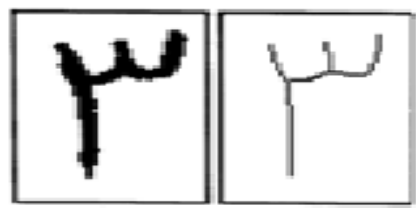

Figure 1: Digit 3, before and after narrowing phase

Using clustering process, pen movement path is recognized and the specified patterns are stored in the learning phase. It is clear that identifying the movement path is not necessary for online inputs.

In other study, the contrast gradient of points has been used to recognize Latin handwritten digits [6]. In this method, normalization is the major preprocess before creating contrast gradient. Normalization eliminates the noises of images. Then, original images are converted to two color images (that means all gray levels are converted to white or black). Next, the dimensions of images are changed to $40 * 40$ pixels.
Finally, the contrast gradient is sent to a neural network to recognize. In [4] handwritten digits recognizing is made by frequency of black and white points of the normal images that have been created in learning phase. After normalization the image size to $40 * 40$ pixels, 300 instances of each digit as $40 * 40$ matrices are added together. Thereafter, a matrix is created for each digit as a pattern which its entries are values in $(-300,300)$. It is because the entries of matrices are 1 for black points and -1 for white points. To recognize a new input handwritten digit, it is compared with all the patterns to choose the one which has the maximum similarity with new instance.

The major goal of this research is to introduce an efficient method to recognize Persian handwritten digits and letters that is written by a specified person. Specifically, this article proposes a new method to recognize Persian handwritten digits and letters through narrowing, clustering, and movement path identification which are introduced in [6]. At first, we specify the principal points of input image and their sequences. Output of this process is a sequence of discrete points and is used to compare with the patterns. For example, digit 3 after two above processing is converted to chain code 75750 that contains five vectors (see figure 2a). Directions of template vectors are shown in figure $2 b$.

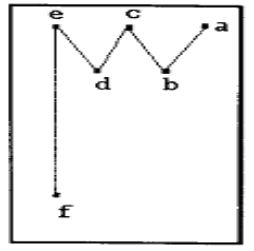

(a)

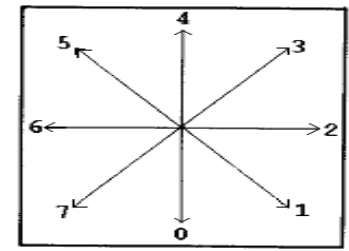

(b)
Figure 2: Principal points of digit 3 and its movement path (abcdef)

Next, the slope and curvature values of input image are compared with all of the patterns to choose the most relevant. In next paragraph vector curve expression is introduced.

\section{VECTOR CURVE}

Suppose that the set of points $\mathrm{P}_{0}, \mathrm{P}_{1}, \mathrm{P}_{2} \ldots$ and $\mathrm{P}_{\mathrm{n}}$ are given in Cartesian space, set of vectors $\mathrm{P}_{0} \mathrm{P}_{1}, \mathrm{P}_{1} \mathrm{P}_{2} \ldots$ and $\mathrm{P}_{\mathrm{n}-1} \mathrm{P}_{\mathrm{n}}$ is called a vector curve on specified points. $\mathrm{P}_{0}$ and $\mathrm{P}_{1}$ are the first and last points and $\mathrm{P}_{0} \mathrm{P}_{1}$ is the first vector of the curve. In figure 3 , digit 5 is shown by a vector curve.

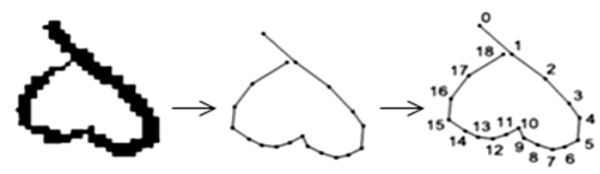

Figure 3: Vector curve of digit 5 


\subsection{Slope of vector curve}

For each vector curve $C$ with set of points $\mathrm{P}_{0}, \mathrm{P}_{1}, \mathrm{P}_{2} \ldots$ and $\mathrm{P}_{\mathrm{n}}$ which $\left(\mathrm{X}_{\mathrm{Pi}}, \mathrm{Y}_{\mathrm{pi}}\right)$ is Cartesian coordinate of $\mathrm{P}_{\mathrm{i}}$, the slope function of each point $\mathrm{P}_{\mathrm{i}}$ on $\mathrm{C}$ must be satisfied in equations 1and 2:

$$
\begin{aligned}
& x_{i}=x_{i-1}+d\left(P_{i}, P_{i-1}\right)=x_{i-1}+\sqrt{\left(x_{P_{i}}-x_{P_{i-1}}\right)^{2}+\left(y_{P_{i}}-y_{P_{i-1}}\right)^{2}} \\
& \text { and } x_{0}=0 \\
& y_{i}=\frac{y_{P_{i}}-y_{P_{i-1}}}{x_{P_{i}}-x_{P_{i-1}}} \quad \text { and } y_{0}=y_{1}
\end{aligned}
$$

Where $d$ is Euclidean distance of two points $P_{i}$ and $P_{i-1}$. Figure 4 shows the slope functions of vector curve of digit 5 that is presented in figure 3. X-Axis unit of figure 4 is in pixel.

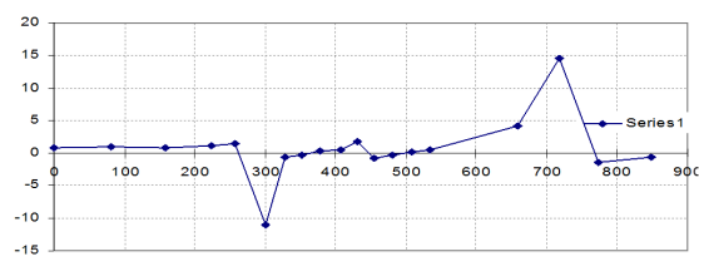

Figure 4: Slope functions of digit 5

\subsection{Curvature of vector curve}

For each vector curve $C$ with set of points $\mathrm{P}_{0}, \mathrm{P}_{1}, \mathrm{P}_{2} \ldots$ and $\mathrm{P}_{\mathrm{n}}$ which $\left(\mathrm{X}_{\mathrm{Pi}}, \mathrm{Y}_{\mathrm{pi}}\right)$ is Cartesian coordinate of $\mathrm{P}_{\mathrm{i}}$, the curvature function of each point $\mathrm{P}_{\mathrm{i}}$ on $\mathrm{C}$ must be satisfied in equation 3:

$\left\{\begin{array}{l}x_{i}=x_{i-1}+d\left(P_{i}, P_{i-1}\right) x_{i-1}+\sqrt{\left(x_{P_{i}}-x_{P_{i-1}}\right)^{2}+\left(y_{P_{i}}-y_{P_{i-1}}\right)^{2}} \\ y_{i}=f\left(P_{i-1}, P_{i}, P_{i+1}\right)\end{array}\right.$

and $x_{0}=0 \quad$ The $\mathrm{y}_{\mathrm{i}}$ is the curvature value at point $\mathrm{P}_{\mathrm{i}}$.To calculate the curvature of $\mathrm{C}$ at point $\mathrm{P}_{\mathrm{i}}$, first the perpendicular bisectors of $\mathrm{P}_{\mathrm{i}-1} \mathrm{P}_{\mathrm{i}}$ and $\mathrm{P}_{\mathrm{i}} \mathrm{P}_{\mathrm{i}+1}$ are identified. Then the distance between $\mathrm{P}_{\mathrm{i}}$ and the cross point of two perpendicular bisectors is named as $r$. The curvature value at point $P_{i}$ will be equal to $1 / r$.

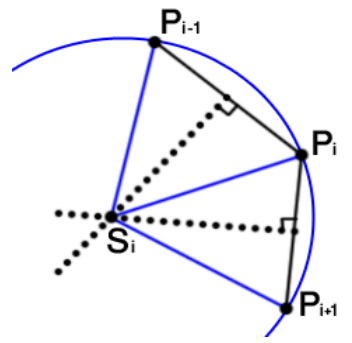

Figure 5: Calculation the curvature value at point $p_{i}$

Equation 4 is used to extract the line equation between two points $\mathrm{P}_{\mathrm{i}}$ and $\mathrm{P}_{\mathrm{i}-1}$. Consequently, equations 5 and 6 compute the perpendicular bisectors of two vectors $\mathrm{P}_{\mathrm{i}-1} \mathrm{P}_{\mathrm{i}}$ and $\mathrm{P}_{\mathrm{i}} \mathrm{P}_{\mathrm{i}+1}$ as:

$$
\left(y_{i}-y_{i-1}\right) x-\left(x_{i}-x_{i-1}\right) y-x_{0}\left(y_{i}-y_{i-1}\right)+y_{0}\left(x_{i}-x_{i-1}\right)=0
$$

$$
\begin{aligned}
& \left\{\begin{array}{l}
x=A_{i} t_{i}+\frac{x_{P_{i-1}}+x_{P_{i}}}{2} \\
y=B_{i} t_{i}+\frac{y_{P_{i-1}}+y_{P_{i}}}{2}
\end{array}\right. \\
& \left\{\begin{array}{l}
x=A_{i+1} t_{i+1}+\frac{x_{P_{i}}+x_{P_{i+1}}}{2} \\
y=B_{i+1} t_{i+1}+\frac{y_{P_{i}}+y_{P_{i+1}}}{2}
\end{array}\right.
\end{aligned}
$$

By initialization the values $\mathrm{x}$ and $\mathrm{y}$ of equation 5 by equation 6 and resolve the equation 7 in the case that the determinant of matrix $\left[\begin{array}{ll}A_{i} & A_{i+1} \\ B_{i} & B_{i+1}\end{array}\right]$ is non-zero, the cross point of two perpendicular bisectors is obtained that is called as $\mathrm{S}_{\mathrm{i}}$. The curvature value at $P_{i}$ is equal to $\frac{1}{d\left(S_{i}, P_{i}\right)}$ and $d$ is the Euclidean distance between two points. If the determinant of matrix $\left[\begin{array}{ll}A_{i} & A_{i+1} \\ B_{i} & B_{i+1}\end{array}\right]$ is zero, the curvature value will be considered to zero. In figure 6 , the curvature functions of vector curve of digit 5 are shown. Coordinate axis is in unit of pixel.

$$
\left[\begin{array}{ll}
A_{i} & A_{i+1} \\
B_{i} & B_{i+1}
\end{array}\right]\left[\begin{array}{c}
t_{i} \\
t_{i+1}
\end{array}\right]=\left[\begin{array}{l}
\left(x_{P_{i+1}}-x_{P_{i-1}}\right) / 2 \\
\left(y_{P_{i+1}}-y_{P_{i-1}}\right) / 2
\end{array}\right]
$$

$$
\left[\begin{array}{c}
t_{i} \\
t_{i+1}
\end{array}\right]=\frac{1}{A_{i} B_{i+1}-B_{i} A_{i+1}}\left[\begin{array}{cc}
B_{i+1} & -A_{i+1} \\
-B_{i} & A_{i}
\end{array}\right]\left[\begin{array}{l}
\left(x_{P_{i+1}}-x_{P_{i-1}}\right) / 2 \\
\left(y_{P_{i+1}}-y_{P_{i-1}}\right) / 2
\end{array}\right]
$$

$$
t_{i}=\frac{B_{i+1}\left(x_{P_{i+1}}-x_{P_{i-1}}\right)-A_{i+1}\left(y_{P_{i+1}}-y_{P_{i-1}}\right)}{2\left(A_{i} B_{i+1}-B_{i} A_{i+1}\right)}
$$

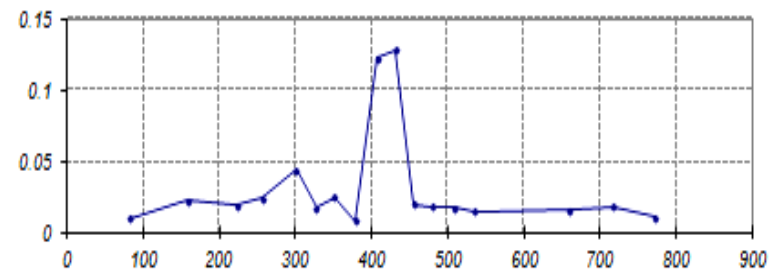

Figure 6: Curvature functions of digit 5 
Curvature and slope functions explain some characteristics of vector curves. These characteristics are Euclidean distance between the points, the state of two neighborhood points (slope) and three contiguous points (curvature).

\section{PRE-PROCESSING}

Before drawing slope and curvature functions, we perform a sequence of proposed pre-processing which are shown in figure 7. First, the image noises are removed in smoothing phase. In normalizing phase, the image scaling will be applied a uniform size to all input samples. Hence, the undesirable effect of various scales will be deleted. Afterward, the fuzzy techniques are used by narrowing [2] and clustering [7] phases to identify the principal points of images and movement paths. Finally, we use curvature and slope functions to store some characteristics of images.

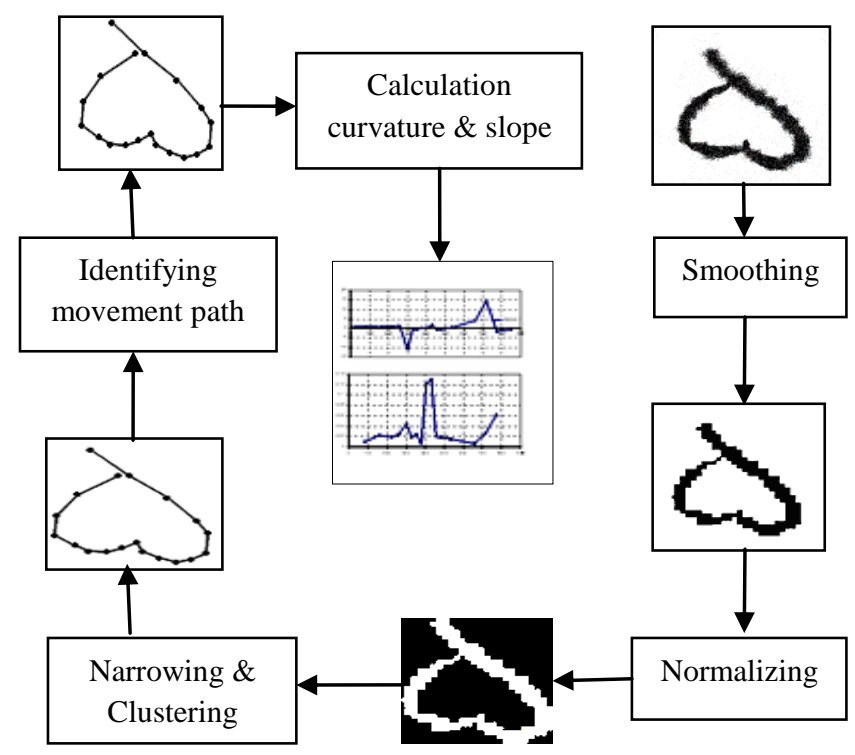

Figure 7: Pre-processing phases

\section{PATTERN CREATION}

We choose a set of scanned handwritten randomly. After initial pre-processing, slope and curvature functions of the samples are created. Drawing the slope and curvature functions attain to the images like figure 8 . The figure shows that the curves are not overlapped, but the sequences of corresponding points in different samples are similar. Furthermore, the points have a normal distribution with $99 \%$ confidence interval in both the $\mathrm{x}$-axis and $\mathrm{y}$-axis. In figure 8 , the confidence interval of a part of slope functions of digit 5 is represented as a box.

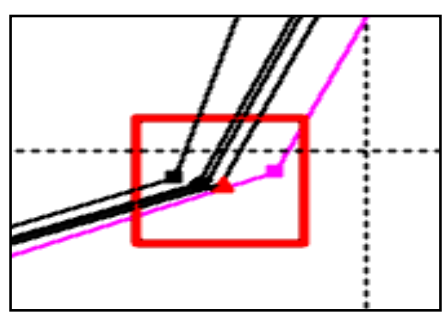

Figure 8: Pattern creation using slope function of samples

\section{EVALUATION THE PROPOSED METHOD}

After creating the patterns, any input image is compared with all available stored patterns. If all of the curve's points lie within the confidence interval of a special pattern, the pattern is selected as output of recognition algorithm. Two problems that may be happened are:

First, no stored pattern is specified with above conditions and second, more than one pattern may be selected. To solve these problems, we define and use a similarity function that is shown in equation 10 .

$$
f(I, P)=1 / \sum_{i=1}^{n} d\left(I_{i}, P_{i}\right)
$$

Where:

I: Slop or curvature function of input image,

P: Slop or curvature function of pattern,

$I_{i}: i^{\text {th }}$ point of $I$,

$P_{i}: i^{\text {th }}$ point of $P$,

$\mathrm{d}\left(\mathrm{I}_{\mathrm{i}}, \mathrm{P}_{\mathrm{i}}\right)$ : Euclidean distance between two points.

We collected 1000 handwritten instances for each letter and digit. In total, 10000 samples for digits and 32000 samples for alphabet letters were prepared. $20 \%$ of samples were used to create the patterns and the rest of them were used for test. All instances were taken from only one person handwritten. Table 1 shows the results of the tests.

Table 1: Percent of correct results

\begin{tabular}{|l|c|c|c|}
\hline \multicolumn{1}{|c|}{$\begin{array}{c}\text { Proposed method with different } \\
\text { conditions }\end{array}$} & Digits & Letters & Average \\
\hline Match to the confidence interval & 69.56 & 46.12 & 51.70 \\
\hline $\begin{array}{l}\text { Match to the confidence interval \& } \\
\text { Use the absolute value of the slopes }\end{array}$ & 97.64 & 92.87 & 94 \\
\hline $\begin{array}{l}\text { Match to the confidence interval \& } \\
\text { Use the absolute value of the slopes } \\
\text { with the similarity function }\end{array}$ & 99.13 & 96.31 & 96.98 \\
\hline
\end{tabular}

\section{CONCLUSION}

Handwritten recognition systems are used in many fields and various methods have been proposed which each of these methods is based on certain techniques to analyze and recognize handwritten characters. The proposed methods in this paper use the geometrical computing and fuzzy techniques. After the preprocessing phases, the images of samples are converted to vector curves. To create and identify the patterns, we used the slope and curvature features of vector curves which in spite of the small number of instances, the results were acceptable. 


\section{REFERENCES}

[1] J. Dong,"Comparison of Algorithms for Handwritten Numeral Recognition", Center of Pattern Recognition and Machine Intelligence, Concordia, pp.1-19, 1999.

[2] R.C. Gonzalez and P. Wints, "Digital Image Processing", Addison-Wesley pp. 399-402, 1987.

[3] H. Ishibuchi, K.Nozaki and H. Tanaka, "Distributed Representation of Fuzzy Rules and its Application to Pattern Classification", Fuzzy Set and System, Vol. 52, pp. 21-32, 1992.

[4] H. Khosravi and E. Kabir, "Introducing a Very Large dataset of handwritten Farsi digits and an study on their varieties" Center of Pattern Recognition Letters Vol. 28, pp. 1133-1141, 2007.

[5] C.L.Liu, et al,"Handwritten Digit Recognition: Benchmarking of State-of-the-art Techniques", Pattern Recognition, pp.2271-2285, 2003.

[6] M. shi et al,"Handwritten Numeral Recognition Using Gradient and Curvature of Gray scale Image", Pattern Recognition, pp.2051-2059, 2002.

[7] P.siy and C. S. Chen "Fuzzy Logic for Handwritten Numerical Characters Recognition", IEEE Trans. Sys. Man. Cybern, pp.570-575, 1974. 